\title{
TuRISMO DE SALUd COMO DINAMIZADOR DE LA ECONOMÍA DE MEDELLíN
}

\section{Tourism of health as dynamizer of the economy of Medellin}

\author{
Luiz Vicente Ovalles Toledo ${ }^{1}$, Carlos Eduardo Llanos Goenaga ${ }^{2}$, \\ Ivonne Esther Acendra Henríquez ${ }^{3}$
}

Recibido: Febrero 23 de 2016/Aceptado: Mayo 26 de 2016

\begin{abstract}
RESUMEN
El presente artículo analiza el auge del turismo de salud en el contexto internacional y su desarrollo y consolidación como actividad económica en la ciudad de Medellín, como servicio con una de las mayores ofertas de este sector de la economía. A través de una recopilación de informes y datos actuales que describen los antecedentes de la industria turística y su recién evidente relación con el segmento de la salud, se evidenció la competitividad de la economía nacional y local en este sector específico. En esa línea, se logra establecer que el turismo de salud en la economía medellinense es un factor en crecimiento, determinante y oportuno como modelo de negocio actual y futuro. El escrito finaliza con algunas reflexiones sobre el debate entre necesidad y ocio de esta industria y los aportes que puede brindar a la economía antioqueña si continúa con el crecimiento y calidad que atrae la atención del público extranjero.
\end{abstract}

Palabras clave: Turismo de salud, Turismo médico, Economía, Movilidad internacional, Competitividad.

\section{ABSTRACT}

This article analyzes the rise of health tourism in the international context and its development and consolidation as an economic activity in the city of Medellín, as a service with one of the largest offers in this sector of the economy. Through a compilation of current reports and data that describe the background of the tourism industry and its newly evident relationship with the health segment, the competitiveness of the national and local economy in this specific sector was evident. In this line, it is possible to establish that health tourism in the economy of Medellín is a growing, determining and opportune factor as a current and future business model. The paper ends with some reflections on the debate between need and leisure of this industry and the contributions it can provide to the economy of Antioquia if it continues with the growth and quality that attracts the attention of the foreign public.

Keywords: Health tourism, Medical tourism, Economy, International mobility, Competitiveness.

Cómo referenciar este artículo: Ovalles, L., Llanos, C. \& Acendra, I. (2016). Turismo de salud como dinamizador de la economía de Medellín. Ad-Gnosis, 5(5), 57-69.

1. Licenciado en Administración de Empresas. Doctor en Administración. Profesor Investigador de la Facultad de Contaduría y Administración, Universidad Autónoma de Sinaloa. Líder del Cuerpo Académico en Consolidación Gestión Estratégica Facultad de Contaduría y Administración FCA UAS, México. luiz.ovalles@uas.edu.mx

2. Docente de la Institución Universitaria CEIPA (Nodo Barranquilla). Administrador de Empresas. Maestría en Administración de Empresas e Innovación. carlos_llanosgo@virtual.ceipa.edu.co

3. Docente, Universidad Simón Bolívar. Ingeniera de Mercados. Maestría en Desarrollo y Gestión de Empresas Sociales. ivonneacendra07@gmail.com 


\section{Introducción}

El turismo como actividad económica asciende en un contexto global que ofrece múltiples opciones para viajeros que buscan diversificar sus actividades y para países que amplían su portafolio de servicios, incentivando la economía a partir de la competencia y la calidad. En este sector, el turismo de salud destaca por una amplia oferta para los usuarios y la innovación en lugares para prestar los servicios, situación que ha tenido como consecuencia una firme implementación de este novedoso pilar en la industria turística de países como Estados Unidos, España o México, y por supuesto, Colombia.

Para establecer la influencia de esta actividad económica en la economía local es pertinente realizar un análisis descriptivo de su evolución histórica en el mundo, que permita conocer cómo Medellín se convirtió en un referente en turismo de salud, de manera que esta reflexión forme parte de los diferentes insumos académicos para el diagnóstico acertado del comportamiento de los sectores económicos y a su vez, reduzca la incertidumbre en los emprendedores y mejore la calidad de la información que se procesa día a día en los mercados mundiales.

El artículo se propone consolidar la información de la caracterización particular de este mercado para concluir, a partir de la lectura, que Medellín contribuye a mejorar la competitividad del país en este nicho y el crecimiento de su economía en general. Aunque esta pro- puesta no busca dar respuestas absolutas ni brindar estrategias comerciales, a través de la descripción se centra en el análisis objetivo del contexto nacional e internacional, determinando la influencia y condiciones futuras del objeto de estudio para el desarrollo económico de Colombia.

\section{Recorrido histórico y conceptual del turismo de salud}

La dinámica del turismo de salud en Colombia como dinamizador de la economía ha cogido mayor fuerza durante los últimos años, este representa un gran porcentaje económico para Medellín. Hoy por hoy, los avances tecnológicos y científicos ayudan y brindan a las personas infinidades de alternativas para su salud y bienestar, por lo cual las personas no pasan desapercibidas; investigan y viajan con el fin de que les ofrezcan mejores soluciones a sus problemas de salud, figura que los convierte en últimas en "turistas de salud".

Hoy día las personas son conscientes de la importancia de llevar una buena vida saludable, las tecnologías de la información y comunicación han ayudado mucho, ya que en la mayoría de las propagandas de televisión sacan anuncios con relación al cuidado y bienestar que se debe tener con respecto al tema, campañas publicitarias con relación a la calidad de vida, eventos, ferias y congresos que incentivan a las personas a mejorar y mantener un excelente estado de salud.

Este turismo que no es muy nuevo para Medellín, trae infinidad de beneficios, entre ellos 
se encuentran los factores económico, social y cultural que otros países puedan traer a este. Las personas se ven motivadas cada día más por las buenas ofertas turísticas de salud que la ciudad puede brindarles, aprovechan esta visita no solo para acceder a los beneficios de salud sino también para conocer e interactuar al mismo tiempo con personas de otros países. Es de gran peso la movilidad e intercambio que estas personas traen a la ciudad, por ello para responder a la demanda y necesidad del cliente, Medellín se esfuerza en tema de innovación y competitividad.

Un fenómeno social que consiste en el desplazamiento voluntario y temporal de los individuos o grupos de personas generando múltiples interrelaciones de importancia social, económica y cultural. (De la Torre Padilla, citado por Arias, Pallares \& Matos, 2012, p.3)

Es importante aclarar, que el sistema de turismo de salud es categoría exclusiva solo para personas que logren permanecer en la ciudad por un tiempo inferior a un año, pues es así como lo establece la Organización Mundial del Turismo (OMT, 2016).

El tema de turismo toca diferentes aspectos, en este caso el más importante es el tema de salud, esta tendencia ha crecido más que la gran mayoría de personas tanto nacionales como internacionales se preocupan solo por llevar un buen aspecto físico sino también mental y espiritual, por ello buscan alternativas para cuidar y prolongar sus vidas.
Los cambios de estilo de vida y deseo de mantener cuerpo y mente sanos son una tendencia general que atraviesa raza, clase, género y edad (...) este fenómeno se ha convertido en una profesión o religión, con el turismo como uno de los medios y fines principales. (Jafari, 2005)

La salud en Medellín es definido como "un estado completo del bienestar físico, mental, social, no corresponde solamente a la ausencia de una enfermedad" (Organización Mundial de la Salud, 2013). Por otro lado los autores definen que en cuanto a turismo de salud, este se refiere a "el desplazamiento o acto de viajar a un lugar determinado para la realización de alguna intervención quirúrgica" (The Tourism Observatory for Health, Wellness and Spa, 2013). Es importante tener claridad que las personas no solo viajan a otros países con la intencionalidad de realizarse una operación quirúrgica e irse a su país de origen, tambien lo hacen para recibir tratamientos con medicina alternativa o porque simplemente encuentran un mejor precio en los servicios y productos.

Con el fin de encontrar e investigar cuáles son los principales servicios de salud por los cuales los turistas prefieren acudir a otro país, se encontró lo siguiente:

1. Medicina curativa: busca eliminar una enfermedad o condición médica desfavorable preexistente y tratar una condición médica específica, promoviendo la buena salud de las personas. Los tratamientos y procedi- 
mientos más sobresalientes son el manejo de artritis, dolores crónicos, trasplantes, cirugía vascular, entre otros.

2. Medicina preventiva: busca prevenir futuras apariciones de enfermedades, optimizando la salud a través de transiciones. Los tratamientos más sobresalientes son los chequeos ejecutivos, tratamientos de traumatologías ortopédicas de baja complejidad, entre otros.

3. Medicina estética: busca la satisfacción de la apariencia física. Los tratamientos más sobresalientes son las cirugías plásticas con fines estéticos (mamoplastia, rinoplastia y liposucción, tratamientos odontológicos, acné y soluciones a desórdenes de pigmento, tratamiento de belleza alternativo, botox).

4. Bienestar (Inspired wellness): busca la satisfacción emocional de los pacientes a través del énfasis social, espiritual y físico/ ambiental. Este campo se ha desarrollado a partir de la necesidad del manejo del estrés y el sobrepeso. Los tratamientos más sobresalientes son la exploración por medio de la meditación, talleres de nutrición, programas de bienestar para toda la familia, talleres de estilo de vida (De la Puente, 2015, pp.134-135).

Este mecanismo implementado en Medellín, hace que se convierta en un negocio más para los servicios que ofrece la ciudad, que la economía se incremente con las nuevas e innovadoras formas de ofrecer salud, lo que se convierte en un componente positivo ya que responde a las demandas y restos de otros países, permite que la ciudad esté en una constante actualización en sus procesos de salud y de servicios a favor del turista, ya que como se mencionó anteriormente este debe permanecer al menos un año en la ciudad, lo que hace que tenga que invertir en hospedaje y alimentación durante su estadía. Otra definición de turismo de salud, García-Altes, citado por Arias et al. (2012) "las formas en las cuales los pacientes viajan a otros países en la búsqueda de una mejoría de la salud" (p.3), es decir, las personas viajan con la finalidad de sentirse mejores consigo mismo.

La mayor cantidad de personas que viajan a la ciudad de Medellín, son principalmente de Estados Unidos, estas personas buscan más que todo la medicina de bienestar, que como se explicó anteriormente, es la que ofrece a las personas una medicina libre de intervenciones y químicos. Estos turistas se ven seducidos tanto por la gran cantidad de opciones que tienen, como por el precio y diversidad de fauna y flora con la que cuenta Medellín.

Continúan con la medicina natural por la cual muchos turistas se ven interesados, está por ejemplo: centros de relajación como Spas, centros de salud innovadores de sal ( medicina natural), acupuntura, terapias biológicas, esquemas sobre la mente y el cuerpo, fisioterapia, aromaterapia, pilates, entre otros, relacionados con la salud mental del paciente. Por 
el contrario varios autores conciben el turismo de salud como: "La realización de intervenciones médicas y médico-quirúrgicas en lugares diferentes al de residencia" (Arias et al., 2012, p.4). Son muchos los turistas que viajan por motivos de salud y cada uno encuentra en la medicina diferentes alternativas para disfrutar de este servicio.

En efecto, son muchos los servicios que ofrece la medicina en Medellín para el bienestar y tranquilidad de las personas, descrito anteriormente, el servicio de bienestar es uno de los más demandados por los extranjeros, en esta categoría se encuentran servicios tales como: ejercicios mentales y espirituales, juegos recreacionales, música, baile, entre otros ya mencionados. Y como segunda más demandada está la medicina estética, ya que Colombia es uno de los principales países más buscados para este tipo de procedimientos, debido a que muchos de estos turistas consideran que el precio vs calidad no se compara con otros países (Malavera, 2013).

En la investigación llevada a cabo, también se encontró que las personas además de viajar por motivos de bienestar saludable y estético, son muchos los turistas que vienen en busca de sanar alguna enfermedad, cuestión que se sitúa en las principales causas de visita. Smith y Puczkó (2008) hacen una diferencia entre personas enfermas y sanas: "Aunque los primeros pueden relajarse y disfrutar del proceso de recuperación mediante el viaje a otro lugar, las motivaciones no son las mismas para los pacientes sanos, que simplemente buscan disfrutar de su condición actual” (p.41).

Trayendo a colación nuevamente la medicina alternativa, los turistas buscaban actividades con relación a la medicina curativa de forma natural:

En las primeras civilizaciones como India, China, Mesopotamia y Egipto, el turismo de salud o turismo médico, se presentó en mayor medida como la peregrinación de las personas hacia los templos sagrados para tomar baños medicinales y aguas termales. Alrededor del 4000 a.C., muchas personas realizaban viajes de peregrinación hacia los templos de Mesopotamia para que les diagnosticaran enfermedades, luego se dirigían hacia Sumeria para recibir el tratamiento en centros de salud, los cuales quedaban rodeados de aguas termales. Esta tradición se volvió tendencia hasta principios del siglo XX. (Arias et al., 2012, p.9)

Esta forma de curación no se hizo esperar y llegó a muchos lugares del mundo, el turismo de salud creció sustancialmente, se evidenció que en países como India estas formas de medicina alternativa sí daban resultados y sanaban a la gente, fue tanto el furor e interés por este tema que los científicos se perfeccionaron y hoy día siguen investigando y puliendo este tema. A la vez otros le dan más importancia a la medicina de medicamentos, ya que conside- 
ran que esta puede ser más segura y eficaz en cuanto a dolor se trata. En cuanto a este tema, se debe reconocer que la India fue el primer país que dio a conocer este tipo de medicina empírica. "La cultura hindú ha conservado esa esencia de espiritualidad de las artes curativas (...) en la época de los 60 , el país recibió un gran impulso por la llegada de peregrinos occidentales en busca de profesionales de la medicina alternativa" (Arias et al., 2012, p.11). No se puede decir entonces que la medicina bioenergética es más que la medicina exacta, ya que es evidente que las dos opciones son altamente demandadas por los turistas que viajan a Medellín.

Ahora bien, el paso de la edad media a la edad moderna y Renacimiento, hizo que la medicina de experimento se convirtiera en una ciencia exacta y de mayor confianza para muchas personas. Arias et al. (2012), relata que: "Del siglo XV al XVII en Europa se despertó un interés por la medicina debido a las precarias condiciones sanitarias, lo que hizo florecer el turismo en salud y redescubrir las antiguas técnicas romanas de la curación a través de baños" (p.13). A raíz de esto las personas comienzan a sentir curiosidad por los principios curativos y encuentran las diferentes posibilidades médicas que existieron y que hoy día se siguen conservando. Esta tendencia hizo que los diferentes países como Colombia se interesaran más por investigar en temas de salud de las ciencias exactas como bioenergética.

Estos cambios y transformaciones en la men- talidad de las personas trajo consigo, el turismo constante, menos enfermedades, mejor conciencia en cuanto a alimentación y cuidado físico e interés por una mejor educación en temas de salud. "En Francia por ejemplo, la mejora en la higiene eliminó muchas enfermedades comunes en la región mediterránea, condición necesaria para los flujos turísticos de llegada a finales del siglo XX" (Gordon, 2002 , p.6). Beneficios que no solo permean a las personas de hoy día, sino también a las nuevas generaciones que vienen.

Los países más recurridos por las personas en búsqueda de una mejor calidad de vida eran: Europa, Holanda y Gran Bretaña, y más específicos a la medicina curativa: India, Suiza, Rusia y Estados Bálticos (Voigt \& Pforr, 2014, pp.205206). Hoy día Colombia compite entre los países más apetecidos por los turistas de salud.

En cuanto a edades los expertos opinan: "los rangos de edad de la población parecen estar cambiando, a principio de la década el público extranjero turista superaba los 30 años, en los últimos años la tendencia de este rango se ha ampliado" (Organización Mundial del Turismo, 2016). Lo que quiere decir sin importar la edad, las personas consideran este tema primordial en sus vidas.

Con la llegada de la globalización fueron apareciendo muchos cambios positivos en cuanto a la calidad de vida, como las Tecnologías de la Información y Comunicación (TIC), el transporte, el intercambio de productos, infraes- 
tructura, etc. Esto trajo a la vez desfavorablemente la aparición de diversas enfermedades en edades tempranas "en América y tras su descubrimiento en 1492, enfermedades como sarampión, lepra y paperas cobraron millones de víctimas entre los aborígenes, puesto que sus sistemas inmunológicos nunca habían estado expuestos a estas infecciones" (Diomedi, citado por Arias et al., 2012, p.14). Los países asiáticos jugaron un papel determinante ya que a pesar de estas nuevas apariciones, lograron demostrar su efectividad en cuanto a la curación natural. "Los aztecas le atribuían propiedades curativas a más de 130 plantas (...), alcanzaron un importante desarrollo en el campo de la cirugía e incluso se dice que el turismo de salud partió de la visita de los colonos desesperados a los curanderos locales como último recurso para aliviar sus enfermedades" (Arias et al., 2012, p.14) experimento que es aplicado en los diferentes países de América y el mundo.

En 2005, la India, Malasia, Singapur y Tailandia, atrajeron más de dos millones y medio de turistas de salud. Colombia, Argentina, México, Cuba, Hong Kong, Hungría, Israel, Jordania, Emiratos Árabes y otros, están en proceso o se han convertido en destinos importantes del turismo médico. (Tata, citado por Arias et al., 2012, p.16)

Calidad y diversidad en sus precios; ofrecen a las personas la posibilidad de tomar estos servicios en vacaciones y descuentos significativos; las pocas regulaciones que existen en torno a este tema y por último los donantes pueden provenir de cualquier parte del mundo, lo cual no es un problema para la personas. Su constancia en el mercado ha sido por motivo de la constante innovación y aprobación que los turistas le dan a estos procedimientos.

Otra de las razones por la cual los turistas viajan es, según la Organización Mundial del Turismo (OMT) "El turismo desde 1998 tiene como principal argumento el ocio, el recreo y las vacaciones constituyendo en un $62 \%$ el motivo principal de viaje de los turistas, seguido de negocios con un $18 \%$ y $20 \%$ para visitas, peregrinajes, salud, etc." (Organización Mundial del Turismo, 2000, p.5). Evidentemente los turistas de salud aprovechan para realizar otras actividades de ocio.

\section{Análisis del desempeño económico de la in-}

\section{dustria internacional, regional y local}

En cuanto a la economía a nivel internacional, regional y local, la Organización Mundial del Turismo (2016) opina: "existe un número creciente de destinos en todo el mundo abiertos al turismo, claves para el progreso socioeconómico, la creación de empleo, la generación de ingresos de exportación y la ejecución de infraestructuras" (Panorama OMT turismo internacional, p.2) el tema del turismo en todos sus aspectos, ha hecho que la economía 
crezca sustancialmente, las personas se han dado cuenta de la alta rentabilidad y avance que este ha traído a sus países. "Las llegadas internacionales a escala mundial pasaron de 674 millones en el 2000 a 1.186 millones en 2015, mientras los ingresos en el mundo incrementaron de $\$ 495.000$ millones en 2000 a $\$ 1$ '260.000 millones en 2015. Hoy, el turismo representa el $7 \%$ de las exportaciones mundiales y ocupa el tercer puesto en las categorías más importantes" (Organización Mundial del Turismo, 2016) aceleración que indiscutiblemente impulsa la evolución y desarrollo de un país.

De acuerdo a investigaciones realizadas, cada país crece en promedio un $5 \%$, pero no es una cifra definitiva ya que son muchos los países que doblan este porcentaje debido a la diversidad de actividades, productos y servicios que ofrecen "De acuerdo a la European SPA Association, Japón tiene el mayor mercado de centros de spa y bienestar en el mundo. En total, los clientes potenciales de estos centros son alrededor de 300 millones en todo el mundo" (p.6). Además "el turismo representa directamente 100 millones de empleos en el mundo, generando un impacto económico que respalda el $9 \%$ del PIB global (\$6.6 billones de dólares en 2012)" (World Travel \& Tourism Council citado por Kazandzhieva, 2014, p.5).

Según el International Medical Travel Journal (2015), "la industria mundial del turismo médico crece hasta en un $25 \%$ cada año a medida que los pacientes con pasaportes se dirigen al extranjero para someterse a procedimientos que cuestan hasta $80 \%$ menos que en sus países" (citado por Giorgadze, 2015, p.21) los turistas ven una gran oportunidad costo - beneficio en otros países, ya que compara con otros países el valor puede ser cinco veces menos que en su país.

Se estima que 11 millones de personas se desplazan cada año de un país a otro con el objetivo de mejorar su salud o aspecto físico, gastando un promedio de 3 mil dólares por viaje, dentro de un mercado mundial con un tamaño aproximado de 15 mil millones de dólares. (Patients Beyond Borders, citado por Giorgadze, 2015, p.21)

Esto se convierte en una gran oportunidad para los países, ya que con ayuda de las TIC, el avance de la ciencia exacta y la medicina natural; los expertos especializados en estos temas podrán sacar todo el provecho que deseen y ofrecer los mejores servicios.

Se estima una tendencia positiva en el crecimiento del sector alrededor del $25 \%$, explicada por el aumento de los servicios médicos en países desarrollados y emergentes, la oferta de profesionales y el aumento de la edad promedio de la población. (Patients Beyond Borders, citado por Giorgadze, 2015, p.21) 
Continuando con las altas cifras de viaje y con base al análisis realizado a los 30 países, se observa que el porcentaje aumenta cada vez más en cuanto a oferta y demanda, Canadá es uno de los principales países que se posiciona en los preferidos por los turistas.

Ahora bien, en cuanto a beneficios de precios Vietnam, África y Malasia se encuentran como los más favorables, $\$ 5.352$ dólares y $\$ 7.000$, aunque se debe resaltar que en estos países las personas no encuentran la diversidad de servicios que sí ofrecen en otros espacios. Por otro lado lugares como Costa Rica, Tailandia y Estados Unidos, sí ofrecen diversidad de servicios pero a costos muy altos, los valores oscilan entre \$11.893 y \$ 51.975 dólares, siendo Estados Unidos el valor más alto en tratamientos de cirugía (OCDE, 2005).

Continuando con los resultados y beneficios que ha traído esta tendencia, entre los años 2011 y 2012 se registró 21.868 turistas en España en búsqueda de tratamientos de salud, alcanzó un gasto de $€ 12,1$ millones de euros (\$14 millones de dólares aproximadamente). (TURESPAÑA, citado por Giorgadze, 2015).

Para la Asociación de la Sanidad Privada Española, "el 51 \% del negocio del turismo de salud se emite desde países europeos, mientras el $32 \%$ desde América. El primer emisor es Estados Unidos, luego siguen Alemania, Francia, Reino Unido, Oriente Medio, Países Escandinavos e Italia" (Lizarralde, 2013, p.99). El porcentaje de comparación entre los países europeos y América Latina es relativamente cercano, posiblemente y en un tiempo no muy lejano, Medellín pueda competir de la mano con grandes potencias.

Según la Organización Mundial del Turismo (OMT) y la OCDE (2005), los valores de los tratamientos en España son de \$4.205 dólares, situándose por debajo del promedio mundial. Los tratamientos más costosos son: reemplazo de cadera (\$15.261 dólares), reemplazo de rodilla (\$15.979 dólares) y estiramiento facial (\$8.561 dólares). Los implantes de senos, demandada operación a nivel mundial tienen un costo de $\$ 6.743$ dólares. Mientras que, las cirugías de menor costo son: implantes dentales (\$1.532 dólares) y liposucción (\$3.876 dólares).

Al igual que muchos otros países, México se destaca por ser también el preferido de los turistas, ya que una de las razones es porque la mayoría de los hospitales y centros de servicio de salud cuentan con acreditación por la Joint Commision International $(\mathrm{JCl})$, calificación que los pone por arriba de muchos otros países. Con base a resultados de ProMéxico (2013) "para 2013 el total ingresos estimados fue de \$2.847 millones de dólares en este segmento del turismo, con lo cual, se proyecta una tasa de crecimiento de 7,3 \% anual" (p.3) y "para 2015 unos 450.000 visitantes del mercado norteamericano fueron atraídos por los servicios médicos de México, lo que dejó unos ingresos estimados de $\$ 300$ millones de dólares" (Lizarralde, 2013, p.83). 
Un plus que se le debe dar a este país, es que investigan y trabajan de la mano con clústers médicos quienes exportan sus servicios a diferentes países del mundo ProMéxico (2013): "Alrededor de 10 estados del país tienen clústeres agrupados en 70 hospitales. Además existen otros hospitales y clínicas que aunque no están agrupados, proveen servicios de salud a extranjeros" (p.10).

En definitiva lo que hace fuerte a México en comparación con otros países, es que además de ofrecer diversidad en costo-beneficio, la mayoría o todos sus servicios cuentan con certificación internacional, reconocimiento y categoría que da a los clientes confianza y tranquilidad.

\section{Panorama del sector turístico de salud para Medellín y su área metropolitana}

Los extranjeros se ven atraídos a la ciudad de Medellín porque allí además de encontrar buenos accesos a la salud, pueden disfrutar de las distintas actividades y lugares turísticos y emblemáticos que tiene la ciudad; además de lo social y cultural, el valor económico es la principal característica por la que las personas se queden en esta ciudad.

El turismo ha sido uno de los factores primordiales que ha hecho que la economía y los países se expandan cada vez más. Medellín, se ve obligado a perfeccionarse y a renovarse en procesos tanto tecnológicos como científicos, es un reto más que tiene la ciudad pero que de seguro logrará siempre estar entre las ciudades más apetecidas.

A nivel mundial el clúster de salud de Medellín "concentra empresas e instituciones especializadas en medicina, odontología, insumos hospitalarios, tecnología biomédica, telemedicina, producción de medicamentos y desarrollo científico" (Marulanda, 2009, p.46).

Medellín, reconocida por sus grandes proyectos innovadores y por ser una ciudad de empuje en todos los aspectos, ha hecho que muchos de los centros de servicios de salud mejoren en cuanto a infraestructura en hospitales, clínicas, laboratorios y demás; mejora que impulsa a que la ciudad sea reconocida específicamente en este sector de la salud.

Autores como De la Puente (2015) opinan que: "En 2013 en Colombia, estas contribuciones aumentaron $120 \%$ respecto a 2012 y se han caracterizado por brindar servicios de calidad principalmente a pacientes internacionales para fines curativos, preventivos, estéticos o de bienestar integral" (p.143). El hospital Pablo Tobón Uribe invirtió en 2014 cerca de USD\$94,50 millones, la Clínica de Occidente con USD\$80 millones o Hospital Universitario San Vicente de Paúl (reconocido como el primer hospital en realizar un trasplante de doble pulmón y desde entonces ha sido pionero en el tema en todo el mundo) con contribuciones de COP\$12.100 millones. Estas han sido las instituciones médicas mejor calificadas por los colombianos. 
El equipo de la Universidad de Antioquia y el mencionado San Vicente Fundación, han realizado los primeros trasplantes de riñón, hígado, corazón y páncreas de Latinoamérica; y de córnea, médula ósea, laringe, tráquea y otras combinaciones del mundo. (Marulanda, 2009, p.45)

Este reconocimiento y valores financieros invertidos por estas instituciones en temas de salud, posicionan a Medellín como una de las mejores ciudades en este tratamiento.

El Gobierno no es ajeno a todo esto, expresa que las personas de Colombia "perciben a la ciudad como un centro atractivo para el desarrollo de actividades de salud" (Marulanda, 2009, p.46).

Como se mencionó en capítulos anteriores, "EI análisis de las variables geográficas indica que la mayoría de pacientes provienen de Estados Unidos (20\% colombianos residentes), Curazao, Aruba, España, Venezuela, Medio Oriente (Israel), Guatemala, Panamá, Bolivia, República Dominicana, Australia, entre otros" (Fiallo et al., 2010, p.10). Por consiguiente, son muchos los extranjeros que ven la oportunidad de viajar y conocer sobre los servicios de salud.

Ahora, es importante nombrar las razones principales por las cuales las personas extranjeras se motivan por adquirir los servicios $y$ productos de Medellín y conocer por qué las personas toman esta opción Fiallo et al. (2010) a) Economía: este aspecto en comparación con otros países es la que mayor oferta vs calidad ofrece a los turistas de salud; b) Calidad: son conscientes del excelente servicio y compromiso que se tiene con las personas; $c$ ) Seguridad: la confianza, buen nombre y reconocimiento, dan a la persona credibilidad, lo que inspira a poner sus vidas en manos de expertos; d) Ética profesional: el conocimiento y estudio que tenga el profesional entorno al tema de salud, es posiblemente un factor determinante. " 1 de cada 10 personas se entera de Medellín y su oferta de turismo de salud por internet, 7 de 10 por procesos de selección de alguna clínica o recomendaciones por experiencias" (Castaño, 2014, p.29).

\section{Conclusiones}

Medellín es hasta ahora reconocido por sus servicios tanto de cirugía estética como medicina natural, considerado también como turismo de salud. Los turistas han preferido durante los últimos años consultar con especialistas y viajar con el fin de obtener los mejores beneficios, atención, calidad y resultado. La ciudad de la eterna primavera es preferida por millones de turistas extranjeros ya que no solo se ven atraídos por temas como este, sino que aprovechan para conocer diferentes lugares, gente y cultura. Frente a esto, el turismo de salud se convierte en una excelente fuente de ingreso económico para la ciudad.

A raíz de esta tendencia saludable, Medellín ha hecho de su infraestructura lugares cómodos que permitan a las personas disfrutar con cali- 
dad de todos sus servicios tales como: hoteles de alta categoría, hospitales, clínicas especializadas y de alta tecnología, centros de innovación y desarrollo, entre otros que marcan la diferencia con respecto a otros países desarrollados. Todo con el fin de que las personas no visiten la ciudad solo de paso (por salud) sino que también se dejen cautivar por los espacios y actividades que ofrece Medellín.

A modo de conclusión, se ha establecido que el turismo de salud es uno de los principales factores que mayor ingreso tiene la ciudad, en comparación con muchos otros países, Colombia se posiciona entre los preferidos por los turistas extranjeros, esto es un plus que se debe aprovechar y no dejar escapar, por ello, la ciudad debe preocuparse más por innovar y brindar un contexto interdisciplinar que ofrezca a las personas una mayor cantidad de servicios de calidad, innovación y confianza.

\section{Referencias}

Arias Aragones, F., Carabayo Pallares, A. \& Matos Navas, R. (2012). El turismo de salud: Conceptualización, historia, desarrollo y estado actual del mercado global. Clío América, 72.

Castaño, M. A. (2014). Turismo en Salud: Resultados de la sistematización y análisis de las encuestas de satisfacción aplicadas a pacientes extranjeros atendidos en una clínica de Medellín. Revista Intersección, 1(1), 24-35. Tecnología en Organización de Eventos, Politécnico Colombiano Jaime Isaza Cadavid.
De la Puente, M. (2015). Sector del Turismo de Salud: Caso de Colombia. Recuperado de https://ezproxy.americana.edu. co:2099/ehost/pdfviewer/pdfviewer?vid $=1 \&$ sid $=c 5934 d 74-d 847-4717-8 c 3 d-$ 04b8c2890811\%40sessionmgr4009

Fiallo, S. M., Franco, J. C., Jaramillo, C., Mejía, S., Quintero, L. M., Rico, C. \& Márquez Godoy, J. I. (2010). El turismo en Medellín desde la percepción del consumidor como una creciente oportunidad de negocio. Revista Soluciones de Postgrado EIA, (6), 95-107.

Giorgadze, N. (2015). Turismo en Salud. Universidad de Oviedo.

Gordon, B. M. (2002). El turismo de masas: Un concepto problemático en la historia del siglo XX. Historia Contemporánea, 25, 125-156.

Jafari, J. (2005). El turismo como disciplina científica. Política y Sociedad, 39-56.

Kazandzhieva, V. (2014). Trends in the development of spa and wellness tourism. Varna, Bulgaria: University of Economics Varna.

Lizarralde, E. (2013). Turismo de Salud en España. Madrid: Ministerio de Industria, Energía y Turismo.

Malavera Pineda, S. M. (2013). La efectividad de las empresas de Base Social en la ciudad de Medellín. Ad-Gnosis, 2(2), 87-93.

Marulanda Montoya, J. A., Correa Calle, G. \& Mejía Mejía, L. F. (2009). El clúster de salud en Medellín, ventaja competitiva alternativa para la ciudad. Revista EAN, (67), 37-47. 
Organización Mundial del Turismo (OMT). (2000). Datos Esenciales 2000. Datos Esenciales OMT 2000.

Organización Mundial del Turismo (OMT). (2016). Panorama OMT del turismo internacional 2016. Madrid: UNWTO.

Organización Mundial de la Salud (2013). Salud mental: un estado de bienestar. Disponible en: http://www.who.int/features/factfiles/mental_health/es/

Organización para la Cooperación y el Desarrollo Económicos (OCDE) (2005). Recuperado de: https://books.google. com.co/books?id=65DVAgAAQBA$\mathrm{J} \& \mathrm{pg}=\mathrm{PA} 3 \& \mathrm{lpg}=\mathrm{PA} 3 \& \mathrm{dq}=$ Organizaci\%C3\%B3n+para+la+Cooperaci\%C3\%B$3 n+y+e l+$ Desarrollo + Econ $\% C 3 \% B-$ $3 \mathrm{micos}+(\mathrm{OCDE})+(2005)$. \& source $=-$ bl\&ots=Dc10POsJ19\&sig=sAngyf\&iEco6Js3hjUwwntsuv3c\&hl=es\&sa=X\&ved=0ahUKEwi67ND2nazcAhXppVkKHR6TBqcQ6AEIZzAH\#v=onepage \&$q=$ Organizaci\%C3\%B3n\%20para\%20 la\%20Cooperaci\%C3\%B3n\%20y\%20 el\%20Desarrollo\%20Econ\%C3\%B3micos\%20(OCDE)\%20(2005).\&f=false
Patients Beyond Borders (2015). Treatment \& Country Finder. Obtenido de https://patientsbeyondborders.com/

ProMéxico (2013). Turismo de Salud. México D.F.: PróMéxico: Inversión y Comercio.

Smith, M. \& Puczkó, L. (2008). Health and wellness tourism. New York: Routledge.

The Tourism Observatory for Health, Wellness and Spa (2013). Global Health Tourism Services Grid. Obtenido de http://thetourismobservatory.org/about-tohws/ credo/global-health-tourism-services-grid/

The Tourism Observatory for Health, WeIlness and Spa (2013). Medical Travel and Tourism. Obtenido de http:// thetourismobservatory.org/publications-resources/interviews-professional-books-papers-and-articles-from-tohws/medical-travel-and-tourism/

Voigt, C. \& Pforr, C. (2014). Wellness Tourism: A destination Perspective. New York: Routledge. 
